\title{
MRI Watermarking in the Compressive Sensing Context
}

\author{
Miloš Orović, Tamara Pejaković, Andjela Draganić, Srdjan Stanković \\ Faculty of Electrical Engineering, 81000 Podgorica, Montenegro \\ andjelad@ac.me
}

\begin{abstract}
The paper proposes procedure for the watermarking of the magnetic resonance images (MRI) using Compressive Sensing approach. The watermark is created as a pseudo random sequence, which is embedded into the set of 2D discrete Fourier transform (2D DFT) coefficients of the observed image. The watermarking coefficients are selected along the radial lines in 2D DFT domain. In order to provide successful watermark detection, regardless the radial lineation, the watermark spreading is proposed. The experimental results prove that the proposed embedding procedure provides successful protection of MRI images even in the case when very small number of CS measurements is used for reconstruction. The efficiency of watermark detection is tested after the repeated Compressive Sensing procedure, as well as under the strong noise disturbances.
\end{abstract}

Keywords - Digital watermarking, Compressive sensing, MRI images

\section{INTRODUCTION}

Protection of digital media is intensively studied in many areas of digital signal processing. The digital content should be protected in terms of ownership, authenticity, copyright, etc. In that sense, a signal called watermark is embedded into the original signal. A watermark can be created as a $1 \mathrm{D}$ or $2 \mathrm{D}$ signal, which might be either fragile or robust to different signal processing operations called attacks. The embedding procedure can be done in time, frequency domain or combined time-frequency domain, which depends on the application, signal type and required watermark characteristics [1]-[11].

In this work, we focus on the watermarking approach for robust protection of MRI in biomedical applications. MRI are watermarked mainly to provide security of the content. Also, watermarking in MRI allows private sharing among experts to improve diagnostics [8]. Unlike other digital media, biomedical images are used for diagnostics and therefore MRI needs to be of very high quality. This means that watermark should not introduce any visible degradation, which makes the task more challenging compared to the standard watermarking scenarios in multimedia applications. At the same time, watermark should be robust to different attacks image can be exposed to, such as noise or filtering. Here, a special attention will be devoted to the Compressive Sensing (CS) as a new type of attack. Also, the CS concept will be used for embedding procedure.

CS [12]-[16] has been developed as an alternative way of data sampling and reconstruction and has a growing impact on the digital signal processing, especially in biomedicine. This approach allows high quality reconstruction of sparse signals using a small set of samples. Reducing the number of samples required for signal reconstruction, leads to the lower exposure time to different apparatus and thus lower impact on the patients. Therefore, we analyze the watermarking in the CS scenario, when we deal with a small amount of acquired data, and it influence on MRI reconstruction. Furthermore, since the same CS approach can be repeated on the watermarked image, it may act as an attack influencing the watermark presence. Therefore, we are facing with two challenges: the first one is how to design the CS based watermarking procedure for MRI and to preserve all important information required for diagnostics, while the second is how to make the watermark robust to the CS attack (and other attacks as well). In that sense, the watermarking coefficients in 2D DFT domain are chosen along radial lines used for CS reconstruction. The watermark is created as a pseudorandom sequence along the same radial lines. The CS reconstruction is performed using the TwIST algorithm [17], proving the high quality of MRI and detectable watermark. In order to improve robustness to repeated $\mathrm{CS}$, we proposed the procedure of spreading the watermark onto contiguous radial lines. The results have shown that the watermark remains detectable even after repeated CS, as well under some other attacks such as additive Gaussian and impulse noise.

The paper is organized as follows. Theoretical background on CS technique and algorithm used for CS reconstruction is given in Section II. The overview of the proposed watermark embedding and detection is given in Section III. The experimental results and concluding remarks are given in Sections IV and V, respectively.

\section{COMPRESSIVE SENSING AND IMAGE RECONSTRUCTION}

\section{A. Compressive sensing theory}

Sampling according to the Nyquist criterion is shown not to be necessary condition in sampling signal, in cases when signal satisfies certain conditions. This means that signal acquisition can be done in a random manner with significantly smaller number of samples compared to that by the Shannon-Nyquist theorem. This is the basic concept behind the CS theory. However, in order to be reconstructed using small number of samples, a signal needs to be sparse in certain domain, i.e. represented by a small number of important non-zero coefficients. Reconstruction of the full signal from incomplete set of samples is done using optimization techniques [14], [18]. Let us shortly describe CS procedure. 
Signal $x$ can be represented using the certain transform basis $\Psi$ as:

$$
x=\Psi s,
$$

where $s$ represents transform domain coefficients. If the random selection of the signal samples is performed, the collected samples will form measurement vector $y$ :

$$
y=\Phi x=\Phi \Psi s=\Theta s \text {. }
$$

Matrix $\Phi$ acts as a random sensing matrix. Signal can be reconstructed even if the number of acquired samples $M$ is much smaller that the signal length $N$, i.e. $M<<N$, as long as s is sparse vector. The relation (2) represents undetermined system of equations and needs be solved by using algorithms that includes optimization techniques [14], [16], [18]. One of the commonly used algorithms in image processing is called TwIST and will be described in the sequel.

\section{B. Two-Step Iterative Shrinkage/Thresholding Algorithm (TwIST)}

In applications concerning image reconstruction from the reduced set of samples, gradient based minimization is used (i.e. total variation - TV minimization). Generally, the images do not satisfy sparsity property, neither in spatial domain nor in the frequency domain. However the image gradient expresses sparsity property and therefore can be suitable in minimization problems. In this paper we have used TwIST algorithm, which relies on the iterative shrinkage/thresholding (IST) and iterative re-weighted/shrinkage (IRS) algorithms [17]. It is designed to solve:

$$
\min \left(\frac{1}{2}\|y-\Phi x\|^{2}+\lambda \Im(x)\right)
$$

where $x$ is an image, $y$ is observation vector, $\Phi$ is transform domain matrix, $\mathfrak{I}(*)$ is regularizer and can minimize $l_{l}-$ norm, $l_{0}$-norm, TV semi-norm or similar (in our we use $l_{2^{-}}$ norm) and $\lambda$ is the regularization parameter. Solution is reached by an iterative procedure defined as [17]:

$$
\begin{aligned}
& x_{1}=\Psi_{\lambda}\left(x_{0}+\Phi^{T}\left(y-\Phi x_{0}\right)\right), \\
& x_{t+1}=(1-\alpha) x_{t-1}+(\alpha+\beta) x_{t}+\beta \Psi_{\lambda}\left(x_{t}+\Phi^{T}\left(y-\Phi x_{t}\right)\right),
\end{aligned}
$$

where $t \geq 1, x_{0}$ is the initial vector, $\Psi_{\lambda}$ is denoising operator wich is, in our case, a hard threshold function. Convergence of the algorithm depends on parameters $\alpha$ and $\beta$. Optimal choice of these parameters corresponds to the following relations:

$$
\alpha=((1-\sqrt{\zeta}) /(1+\sqrt{\zeta}))^{2}+1 ; \beta=2 \alpha /(1+\zeta)
$$

where $\zeta$ is set to $\zeta=10^{-1}$ or $\zeta=10^{-3}$ [17].

\section{DIGITAL WATERMARKING OF MRI IMAGES}

Keeping the medical records and information about the patients is very important in medical administration. Using the Internet based data transfer in medical diagnosis, requires secure data protection. In that sense, watermarking of the medical data has become good practice nowadays [8]. As it was mentioned previously, medical content is more vulnerable to the watermark embedding procedure that any other digital media, as preserving all information is of great importance.

\section{A. The watermarking procedure}

The considered MRIs are usually recorded in the 2D DFT domain rather than in the pixel domain. Therefore, the 2D DFT is considered for watermark embedding. The following assumptions are used:

1) In order to speed up the acquisition procedure, only certain lines are sensed in the 2D DFT domain, which are defined by the radial mask $\Lambda$.

2) The important image information is concentrated around the origin in 2D DFT domain, which should be avoided in watermark embedding.

Hence, the watermark is added along radial lines which correspond to the mask formed in 2D DFT domain:

$$
\operatorname{DFT}_{w}^{2 D}(\Lambda)=D F T^{2 D}(\Lambda)+\alpha w
$$

where $D F T$ represents matrix of 2D DFT coefficients used for watermarking, parameter $\alpha$ controls the watermark strength and $w$ is the watermark matrix, formed as:

$$
w=\Theta \Lambda \Upsilon
$$

where $\Lambda$ denotes radial line mask, $\Upsilon$ is random number matrix and $\Theta$ is matrix with zero values in the certain region around the origin. Then the image reconstruction is performed using the TwIST algorithm to prove that the watermark will not influence the image quality. The watermark embedding strength is chosen as a trade-off between the desired quality of reconstructed MRI and the ability to detect the watermark afterwards.

The blind watermark detection procedure is performed by using the standard correlation detector [19], [20]:

$$
R_{w a t}=\sum_{i=1}^{N} w_{i} D F T_{w_{i}} .
$$

The detector response for right key $R_{w a t}$ should be larger than for any other key (wrong key), i.e.:

$$
R_{\text {wrong }}=\sum_{i=1}^{N} \text { wrong }_{i} D F T_{w_{i}}<R_{\text {wat }}
$$

It is assumed that the wrong key is generated in the same way as the right key. The watermark detection is successful as it will be shown in the experimental part, but such watermark can be easily removed by the repeated CS procedure.

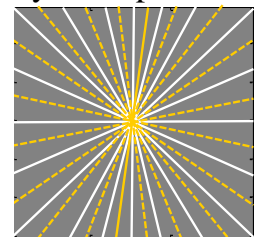

Figure 1: White lines-lines used for watermarking and CS reconstruction, yellow lines-lines without watermark that can be used in reconstruction procedure

Namely the mask $\Lambda$ contains a small number of equidistant radial lines and it is easy to find another combination of radial lines that will have no information about the watermark. White lines in Fig. 1 illustrate watermarked lines in the 2D DFT domain, while yellow dashed lines denote new combination of the lines that do not contain watermark. If these lines are used to reconstruct an MRI again using the 2D 
DFT domain, the watermark will no longer be present in the MRI. In order to overcome this possibility we proposed the watermark spreading procedure.

\section{B. Watermark spreading procedure}

The approach of spreading the watermark along the neighboring radial lines is done in order to improve watermark detection and to assure robustness regardless the lineation of radial lines within the mask. Watermark has been divided into $W$ lines around the original line, with $W$ times lower strength. Therefore, the total number of watermarked 2D DFT lines is now equal to the number of lines within the mask, multiplied with $W$. The watermark is now defined as:

$$
w_{S}=\frac{1}{W} \sum_{k=1}^{W} \Lambda_{k}(l, \theta) \cdot w,
$$

where $w_{S}$ denotes the new watermark, $\Lambda_{k}(l, \theta)$ is radial mask with $l$ lines and $\theta$ denotes angle used for mask rotation. Embedding is done using the same procedure as in relation (12). Watermark is shown in Fig. 2.

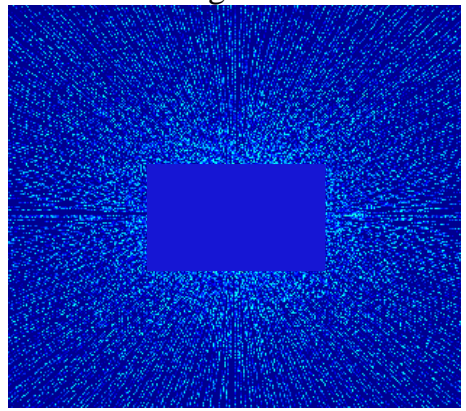

Figure 2: Spreading watermark

\section{NUMERICAL EXAMPLES}

\section{Case 1: 48-lines watermark}

Consider MRI image of $512 \times 512$ coefficients (Fig. 3a). In order to embed watermark, radial line mask is formed and 2D DFT coefficients along the radial lines are chosen for embedding. Mask is formed of 48 lines. Watermark is embedded into the 2D DFT coefficients along radial lines, using additive procedure according to the equation (6). Parameter for watermark strength is $\alpha=1000$. 2D DFT coefficients around the origin $(50 \times 50$ region $)$ are not watermarked in order to avoid watermark to degrade image quality.

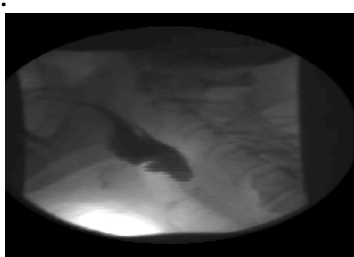

a)

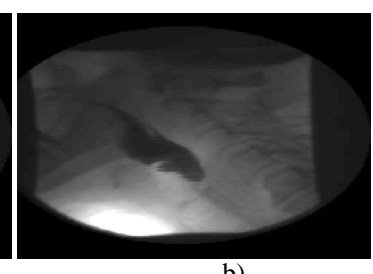

b)
Figure 3. a) Original image; b) watermarked image

Lines used for watermark embedding are, at the same time, used as measurements in CS procedure and image is reconstructed from these measurements using TwIST algorithm. Watermarked/reconstructed image is shown in Fig. 3b. As it can be seen, watermark is imperceptible in the image. Watermark detection is tested using 1000 wrong trials, and detector response is shown in Fig. 4a. Let us test detector response if other 48 lines from the plane are chosen (i.e. mask is rotated for an arbitrary angle). Detector response will be significantly degraded, since the possibility to choose lines that do not contain watermark, is high. Therefore, the correlation of the watermarked coefficients with the watermark will be low and detection will not be reliable as shown in Fig. $4 \mathrm{~b}$.

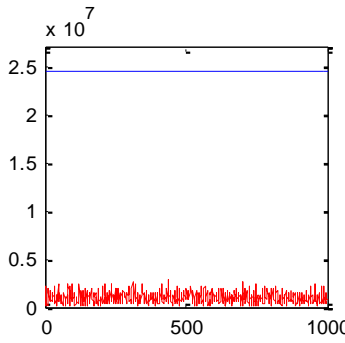

a)

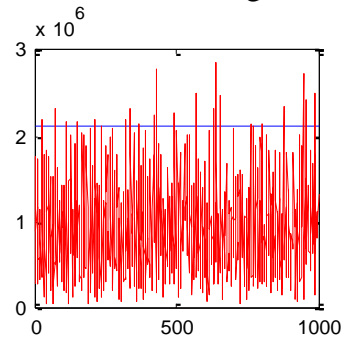

b)
Figure 4. a) detector responses for 48-line mask; b) detector responses with an arbitrary positioned 48-line mask (blue line - right key, red line - wrong trials). Horizontal axis is for trials, vertical axis is for detector response.

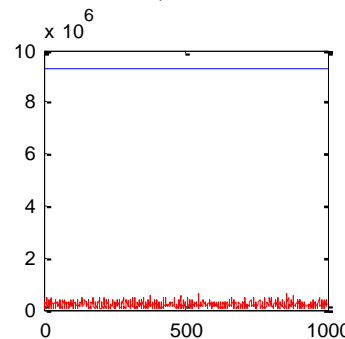

Figure 5. Detector response for spread watermark and arbitrary chosen 48line mask. (blue line - right key, red line - wrong trials). Horizontal axis is for trials, vertical axis is for detector response.

In order to make watermark procedure robust to CS attack, watermark spreading is performed prior to the embedding and it will be described in the sequel.

\section{Case 2: Spreading watermark}

In order to assure watermark detection when 48-line mask is rotated by arbitrary angle, watermark is spread on neighboring lines. We have chosen 4 neighboring lines for spreading, and each line contains $1 / 4$ of the original watermark. Spreading did not affect image quality. Watermarked image is then submitted to CS attack. The same number of radial lines is used for reconstruction (48 lines), but with different positions. On Fig. 5 detector response using 1000 wrong trials and rotated 48-line mask is used. As it can be seen, detector response for the right key is higher compared to the responses using the wrong keys.

Let us now test robustness of the proposed watermark procedure to the several attacks. We have observed noisy image (corrupted by Gaussian and impulse types of noise, with low signal-to-noise ratio), median filtered and JPEG compressed image. Images attacked by Gaussian and impulse noise are shown in Fig. 6. As it can be seen, noise is visible in the image. Even in such cases, watermark can be successfully detected. Detector responses are shown in Fig. 7a and Fig. 7b. Also, detector response is measured in cases when image is median filtered and JPEG compressed. In both cases, 
successful detections are obtained (as shown in Fig. 7c and Fig. $7 \mathrm{~d}$, respectively).

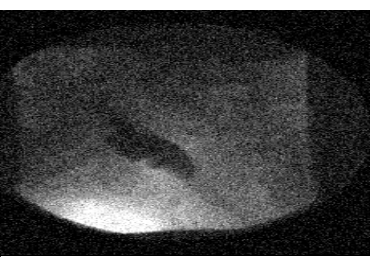

a)

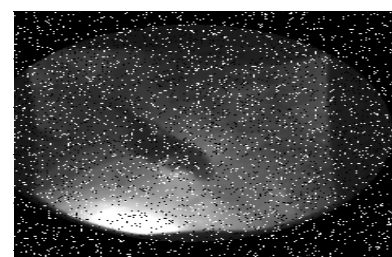

b)
Figure 6. Image corrupted by: a) Gaussian, b) impulse noise $\times 10^{6}$

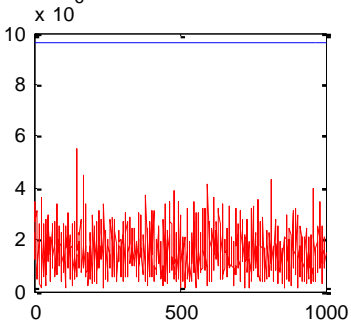

a)

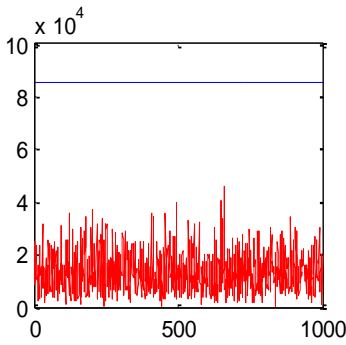

c)

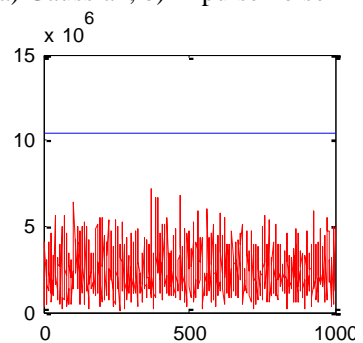

b)

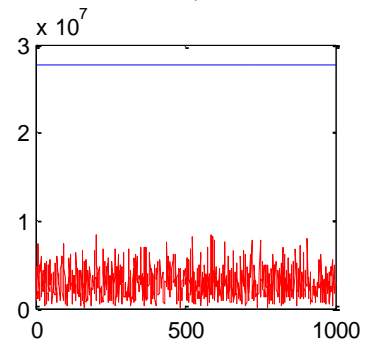

d)

Figure 7. Detector responses for different attacks: a) Gaussian; b) impulse noise; c) median filtering; d) JPEG compression with compression factor $40 \%$. Blue line is for right key, while red line is for the wrong trials.

Horizontal axis is for trials, vertical axis is for detector response.

\section{CONCLUSION}

The MRI watermarking procedure is proposed in the paper. Embedding is done in certain, small number of acquired 2D DFT domain coefficients. Image is then reconstructed from the acquired samples, using CS reconstruction techniques. In order to increase robustness of the proposed watermarking procedure, watermark is spread onto the neighboring lines that belong to the radial mask in 2D DFT domain. It is shown that the watermark detection is successful under CS attack, JPEG compression and median filtering, as well as in the noisy environment.

\section{AKNOWLEDGEMENT}

This work is supported by the Montenegrin Ministry of Science, project grant funded by the World Bank loan: CSICT "New ICT Compressive sensing based trends applied to: multimedia, biomedicine and communications". The authors are thankful to the professor Ervin Sejdić, from the University of Pittsburg, for providing the real biomedical data used in the paper.

\section{REFERENCES}

[1] I. J. Cox, M. L. Miller, and J. A. Bloom, Digital Watermarking. Academic Press, 2002.

[2] S. Stankovic, I. Orovic, and N. Zaric, "Robust watermarking procedure based on JPEG-DCT image compression," Journal of Electronic Imaging, vol. 17, No. 4, Page(s) 043001, 2008.

[3] J. R. Hernandez, M. Amado, and F. Perez Gonzeles, "DCT-domain watermarking techniques for still images: Detector performance analysis and a new structure," IEEE Transactions on Image Processing, vol. 9, No 1, pp. 55-68, 2000.

[4] S. Stankovic, I. Djurovic, and I. Pitas, "Watermarking in the space/spatial-frequency domain using two-dimensional Radon Wigner distribution," IEEE Transactions on Image Processing, vol. 10, No.4, pp. 650-658, 2001.

[5] B. G. Mobaseri, "Digital watermarking in the joint time-frequency domain," IEEE International Conference on Image Processing, New York, vol. 3, pp. 481-484, September 2002.

[6] I. Orovic, P. Zogovic, N. Zaric, and S. Stankovic, "Speech Signals Protection via Logo Watermarking based on the Time-Frequency Analysis," Annals of Telecommunications, vol. 63, No. 5-6, pp. 276284, 2008.

[7] S. Stankovic, I. Orovic, N. Zaric, and C. Ioana, "Two Dimensional Time-Frequency Analysis based Eigenvalue Decomposition Applied to Image Watermarking," Multimedia Tools and Applications, vol.49, No. 3 , pp. 529-543, 2010.

[8] N. O. Abokhdair, and A. Bt Abdul Manaf, "A Prediction-Based Reversible Watermarking for MRI Images," World Academy of Science, Engineering and Technology International Journal of Computer, Control, Quantum and Information Engineering vol. 7, No 2, 2013.

[9] S. Stankovic, "Time-Frequency Analysis and its Application in Digital Watermarking," Review paper, EURASIP Journal on Advances in Signal Processing, Special Issue on Time-Frequency Analysis and its Application to Multimedia signals, vol. 2010, Article ID 579295, 20 pages, 2010.

[10] M. Al-khassaweneh, and S. Aviyente, "A time-frequency inspired robust image watermarking," in IEEE Conference Record of the Thirty-Eighth Asilomar Conference, vol. 1, pp. 392-396, 2004.

[11] E. Muharemagić, and B. Furht, Survey of watermarking techniques and applications. Chapter in Multimedia Watermarking Techniques and Applications, B. Furht and D. Kirovski, editor, Auerbach Publication, pp. 91-130, 2006.

[12] I. Orovic, and S. Stankovic, "Combined Compressive Sampling and Image Watermarking," 55th International Symposium ELMAR 2013, pp. 41-44, Zadar, Croatia, September 2013.

[13] E. J. Candes, and M. B. Wakin, "An Introduction to Compressive Sampling," Signal Processing Magazine, IEEE , vol. 25, No. 2, pp. 2130, March 2008.

[14] S. Stankovic, I. Orovic, and E. Sejdic, Multimedia Signals and Systems. Springer-Verlag, New York, 2012.

[15] M. Lustig, D. L. Donoho, J. M. Santos and J. M. Pauly, "Compressed Sensing MRI," IEEE Signal Processing Magazine,vol. 25, No 2, pp. 7282, March 2008.

[16] S. Stankovic, LJ. Stankovic, and I. Orovic, "Relationship between the Robust Statistics Theory and Sparse Compressive Sensed Signals Reconstruction," IET Signal Processing, Special issue on Compressive Sensing and Robust Transforms, vol. 8, No 3, pp. 223-229, May, 2014.

[17] J. M. Boiucas-Dias and M. A. T. Figueiredo, "A New TwIST: Two-Step Iterative Shrinkage/Tresholding Algorithms for Image Restoration," IEEE Trans. on Image Proc., vol. 16, No 12, pp. 2992 - 3004, November 2007.

[18] P. Boyd, and L. Vandenberghe, Convex Optimization. Cambridge University Press, March 8, 2004 - Business \& Economics - 716 pages.

[19] S. Stankovic, I. Djurovic, R. Herpers, and LJ. Stankovic, "An approach to the optimal watermark detection," AEUE International Journal of Electronics and Communications, vol. 57, No. 5, pp. 355-357, 2003.

[20] A. Nikolaidis and I. Pitas, "Asymptotically optimal detection for additive watermarking in the DCT and DWT domains," IEEE Transactions on Image Processing, vol. 12, No. 5, pp. 563-571, 2003. 